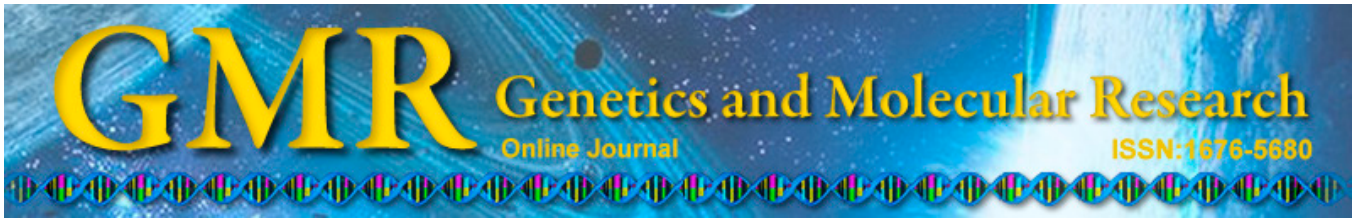

\title{
Clinicopathological analysis of idiopathic membranous nephropathy in young adults
}

\author{
Y. Wang, G.P. Wang, B.M. Li and Q.K. Chen \\ Department of Nephrology, \\ The First Affiliated Hospital of Nanchang University, Jiangxi, China \\ Corresponding author: B.M. Li \\ E-mail: biminlicn@163.com
}

Genet. Mol. Res. 14 (2): 4541-4548 (2015)

Received July 10, 2014

Accepted November 26, 2014

Published May 4, 2015

DOI http://dx.doi.org/10.4238/2015.May.4.12

\begin{abstract}
The aim of this study was to understand the clinicopathological features and prognosis of idiopathic membranous nephropathy (IMN) in youth. A retrospective analysis of the clinicopathological features and prognoses of pathologically confirmed IMN in 21 patients aged 15-30 years was performed. IMN was mainly characterized as nephrotic syndrome (NS), with stage I as the main pathological stage, and associated with hyperplasia of the glomerular mesangial cells and ground substance. High-intensity immunofluorescence also showed multi-site deposition of a variety of immune complexes, and electron microscopy showed multi-site deposition of electron-condensing substances. In the present study, 4 patients received non-specific treatment. Among 17 NS patients, 12 patients exhibited a preference for glucocorticoid therapy, and of these patients, 7 were sensitive to therapy and 5 were resistant. In the 12 patients who received hormone treatment combined with immunosuppressants (including 5 patients who were treated with the combination from the initial start, 5 patients who were steroid resistant, and 2 patients who were sensitive to the initial hormone treatment but who later showed relapse), complete remission was achieved in 6 patients, partial remission was achieved in 2, the treatment was
\end{abstract}


ineffective in 2, and 2 patients were lost to follow-up. In conclusion, the clinical manifestation of IMN in youth in this study was mainly NS. In most patients, the initial hormone treatment was effective, and in some patients, the combination of hormone and immunosuppressant treatment was effective. As the sample size in this study was small, further clinical validation is still required to determine the efficacy of the treatment.

Key words: Glomerulonephritis; Membranous nephropathy; Youth; Nephrotic syndrome

\section{INTRODUCTION}

Idiopathic membranous nephropathy (IMN) is a common histological type of adult nephrotic syndrome (NS), and accounts for approximately 55.7\% of membranous nephropathies, and $9.89 \%$ of primary glomerular disease (Li and Liu, 2004). In previous studies (Ponticelli and Passerini, 2010a), IMN has been reported to account for $25-40 \%$ of adult NS cases. The prognosis of untreated patients is diverse, with $30-60 \%$ of patients exhibiting spontaneous remission. While the majority show improvement, between $34-62 \%$ of patients develop renal dysfunction (Schieppati et al., 1993; Reichert et al., 1998; Glassock, 2003; du Buf-Vereijken., 2005), and approximately $10-30 \%$ of patients progress to end-stage renal disease within 10 years (Waldman and Austin 3rd, 2009).

Typically, IMN occurs in middle-aged and elderly people, with peak age of onset between 31 and 60 years, and a male to female incidence ratio of approximately $2: 1$. In recent years, however, there has been a rising trend in the number of IMN cases in youth. This study, therefore, retrospectively analyzed the clinicopathological features of 21 cases of IMN in younger patients aged between 15 and 30 years old.

\section{MATERIAL AND METHODS}

\section{General information}

Patients were recruited from the Department of Nephrology at the First Affiliated Hospital of Nanchang University between January 2010 and March 2012, and a total of 21 IMN patients aged between 15 and 30 years old were enrolled in the study. Diagnosis was confirmed by renal biopsy, accounting for $4.24 \%$ of the biopsy cases ( 495 cases) of primary glomerular disease during this period. This study was conducted in accordance with the Declaration of Helsinki, and it was conducted with approval from the Ethics Committee of Nanchang University. Written informed consent was also obtained from all participants. Diagnostic criteria for IMN were in accordance with the literature (Xiong et al., 2004) and included light and electron microscopy examinations of renal tissues revealing immune complex deposition on the subepithelium of the glomerular basement membrane (GBM), and diffuse thickening of the basement membrane, as well as, immunofluorescence examinations showing a diffuse and granular pattern of $\operatorname{IgG}$ and $\mathrm{C} 3$ distribution along the basement membrane. Patients with infections (including hepatitis B virus), cancer, systemic lupus erythematosus, and/or drug-induced secondary mem- 
branous nephropathy were, however, excluded from the study. For those included in the study, the following data were obtained: gender, age, blood pressure, urinalysis results, serum creatinine (Scr), serum albumin (Alb), serum cholesterol (Chol), 24-h urinary protein excretion rate, and B-mode ultrasonography of both the kidneys.

\section{Renal biopsy}

According to pathological staging, referring to the staging method in the literature (Ehrenreich and Churg, 1968), the cases were divided into 4 stages: stage I, whereby glomerular structure was normal under the light microscope, vacuolar degeneration could occasionally be seen in the basement membrane, few electron-condensing substances could be seen on the subepithelium under the electron microscopy, while no significant change occurred in the basement membrane; stage II, diffuse thickening of the basement membrane that could be observed under the light microscope, along with the appearance of "rete pegs", and a large number of electron-condensing substances on the subepithelium that could be seen with electron microscopy, as well as a "rete peg"-like proliferated basement membrane causing thickening of the glomerular capillary wall; stage III, a thickened basement membrane with "chain-cycle"-like appearance under light microscopy, diffused thickening of the glomerular capillary wall, stenosis or occlusion of the capillary cavity, slightly increased mesangial matrix, a large number of electron-condensing substances deposited inside the basement membrane that could be visible under the electron microscope, a double-ladder shape, the subepithelial condensing substances partially dissolved; stage IV, thickening of the GBM seen under light microscopy, occlusion of the capillary cavity, an increased mesangial matrix, glomerular atrophy or fibrosis, fused bilayer of the basement membrane, irregular thickening under electron microscopy, a transparent region between the condensing substances, appearance of the basement membrane appear in a chain shape. The fluorescence signal exhibited by the deposition intensity of immunoglobulin could be divided as $(+)$ to $(+++)$.

\section{Treatment methods}

Of the 21 patients included in this study, for 4 patients not meeting the massive proteinuria in NS at disease onset criteria, only angiotensin receptor antagonists were included in the initial treatment, without corticosteroids and immunosuppressive agents. Among them, 2 patients experienced aggravated hematuria and proteinuria. Following initial treatment, a sufficient amount of methyl prednisone $\left(1 \mathrm{mg} \cdot \mathrm{kg}^{-1} \cdot \mathrm{day}^{-1}\right.$; Pharmacia Italia S.P.A., Ascoli Piceno, Italy) was used in subsequent treatments. For 12 patients meeting the massive proteinuria at disease onset criteria, a single treatment with sufficient methyl prednisone was administered. Among them, 7 patients were sensitive to hormone therapy, including 2 patients with hormone resistance after recurrence. In these cases, FK506 (AsteUas Ireland Co., Ltd., Killorglin Co., Kerry, Ireland) was added to the treatment. In the other 5 patients who were not sensitive to hormone therapy, cyclosporine A (IVAX Pharmaceuticals S.R.O, Opava, Czech Republic) was used in 1 patient, cyclophosphamide (Baxter Oncology GmbH, Halle, Germany) was used in 2 patients, and mycophenolate mofetil (MMF) (Roche Registration Ltd., Welwyn Garden City, UK) was used in 2 patients. The remaining 5 patients were treated with corticosteroids combined with MMF from disease onset. 


\section{Statistical analysis}

All statistical analysis was performed using the SPSS 13.0 software. Data are reported as means \pm standard deviation for normal distributions, or median and interquartile interval for non-normal distributions. Categorical variables were expressed as a percentage.

\section{RESULTS}

\section{Clinical manifestations and laboratory results}

The 21 patients with IMN included 16 men and 5 women, with the median age of 20 (range 14 to 29) years old at the time of hospitalization. The median disease duration was 2 (range 0.2 to 60) months when the renal biopsy was performed. The clinical manifestations were 17 diagnosed patients of NS ( 9 cases of simple type and 8 patients of nephritis type) and 4 patients of hematuria and proteinuria, of which 2 patients were accompanied with renal insufficiency where SCr levels were 313 and $144 \mathrm{mM}$, respectively. According to the Schwartz formula, the estimated glomerular filtration rates in these cases were 26.12 and 48.7 $\mathrm{mL} \cdot \mathrm{min}^{-1} \cdot 1.73 \mathrm{~m}^{-2}$, respectively. Additionally, 3 of the 21 patients were accompanied with hypertension (14.3\%). From 21 patients renal biopsies, the average Scr was $79.47 \pm 64.92$ $\mu \mathrm{M}$, the average Chol was $5.83 \pm 3.32 \mathrm{mM}$, and the average Alb was $22.56 \pm 6.15 \mathrm{~g} / \mathrm{L}$. The 24-h urine protein value was $3.43 \pm 1.45 \mathrm{~g}$, and there was no abnormality on the B-mode ultrasonogram of the kidneys, ureter, and bladder, as well as on the color ultrasonogram of the renal arteriovenous fistula.

\section{Light microscopy}

In all study patients, mild proliferation of the glomerular mesangial cells and mesangial matrix was visible. According to the pathological staging criteria, a majority of the cases were classified either as stage I ( 14 cases) or stage II (6 cases), with 1 case in stage III. Among the 21 patients, 2 patients exhibited various degrees of glomerular global sclerosis (the percentages of spherical glomerular sclerotic were 11.1 and 5\%); 4 cases were accompanied with segmental sclerosis (the percentages of the segmental glomerular sclerosis were 16.7, 15, 4.3, and 3\%); 6 cases were accompanied with renal tubular atrophy ( 5 cases were mild, and 1 case was moderate); and 2 cases were accompanied with the fibrous crescent formation of small cells (50 and $11.1 \%)$.

\section{Immunofluorescence}

The immunofluorescence examinations in the 21 patients showed $\operatorname{IgG}$ deposition as the main type $[2$ cases $(+++), 3$ cases $(+), 16$ cases $(++)$ to $(+++)$ ], while 18 cases were accompanied with $\mathrm{C} 3$ deposition [2 cases $(+++), 3$ cases $(+++), 13$ cases $(+)$ to $(++)], 19$ cases were accompanied with IgM deposition $[1$ case $(++++), 2$ cases $(+++), 4$ cases $(++)$, 12 cases $(+)$ ], 14 cases were accompanied with $F g$ deposition [4 cases $(++), 10$ cases $(+)$ ], 18 cases were accompanied with $\mathrm{C} 1 \mathrm{q}$ deposition $[3$ cases $(+++), 5$ cases $(++), 10$ cases $(+)$ ], and 16 cases were accompanied with IgA deposition [ 3 cases $(+++), 2$ cases $(++)$, 11 cases $(+)]$. 


\section{Electron microscopy}

All 21 patients underwent electron microscopy examination, and exhibited extensive fusion, flattening, and microvilli denaturation of the epithelial cell foot process. All the patients also exhibited multi-site deposition of electron-condensing substances, including the subepithelium, subendothelium, mesangial region, and the basement membrane in 3 patients; the subepithelium and mesangial region in 2 patients; the subepithelium and basement membrane in 3 patients; and the subepithelium, mesangial region, and basement membrane in 13 patients.

\section{Treatment results}

Of the 4 patients who did not meet the massive proteinuria in NS at disease onset, 1 patient showed complete remission, 1 showed partial remission, and 2 had aggravated hematuria and proteinuria. For the 2 patients with aggravated conditions, the urine protein converted to negative after treatment with methyl prednisone for 8 weeks. In the 12 patients who met the massive proteinuria at disease onset criteria, a single treatment with sufficient methyl prednisone was administered. Among them, 7 patients were sensitive to hormone therapy, including 2 patients with hormone resistance after recurrence. Of these 2 patients, after treatment with FK506, there was complete remission in 1 patient, and partial remission in the other. In the other 5 patients who were not sensitive to hormone therapy, complete remission was achieved in 1 patient treated with cyclosporine A; of the 2 patients treated with cyclophosphamide, only 1 patient obtained complete remission (the other patient was treated with FK506, and the result was ineffective), and in the remaining 2 patients treated with MMF, there was complete remission in 1 patient while the other patient was lost to follow-up. Finally, of the 5 patients using corticosteroids combined with MMF from disease onset, 2 patients showed complete remission, 1 patient showed partial remission, 1 patient did not respond, and 1 patient was lost to follow-up. Of the total 21 IMN patients, 14 patients showed complete remission, 3 showed partial remission, 2 did not respond, and 2 were lost to follow-up. The overall remission rate was $81 \%$.

\section{Prognosis}

As of September 2012, 2 patients were lost to follow-up, and in 19 patients, follow-ups occurred within 9 and 24 months, with a median follow-up period at 15 months. In 17 patients diagnosed with NS at disease onset, 11 obtained complete remission within 2 to 6 months, while 2 obtained partial remission, 2 did not respond, and 2 were lost to follow-up. Of the 2 patients who experienced renal insufficiency at disease onset, renal function returned to normal in 1 patient, and was restored to $180 \mu \mathrm{M}$ in the other patient but returned to the onset level. Of the 4 patients who did not reach massive proteinuria at onset, and were not administrated glucocorticoid or immunosuppressant therapy, there was complete remission in 1 patient and partial remission in 1 . In the remaining 2 patients, while hematuria and proteinuria increased, the patients finally obtained complete remission following treatment with sufficient hormone $\left[1 \mathrm{mg} \cdot \mathrm{kg}^{-1} \cdot \mathrm{day}^{-1}\right]$.

\section{DISCUSSION}

Typically, IMN occurs in middle-aged men between 31 to 60 years old $(62.6 \%)$, with the main clinical manifestations presenting as edema (80.2\%), NS (52.7\%), hyperten- 
sion $(47.3 \%)$, and microscopic hematuria $(63.7 \%)$, while renal failure $(3.3 \%)$ is a more rare complication. Stages I and II account for $93.4 \%$ of cases, which can also be accompanied by a certain degree of tubulointerstitial and small vascular damage, as well as the deposition of IgG and granular $\mathrm{C} 3$ on the capillary loops and electron-condensing substances on the subepithelium. Currently, there are few reports in the literature regarding the occurrence of IMN in youth. Moreover, the age range for disease onset is also inconsistent between existing reports, with some studies reporting age at onset to be more than 40 years, while others report this to be less than 35 years old (Wu, 2002). Nevertheless, as IMN mainly occurs between the ages of 31 and 60 years, patients younger than 30 years old in the present study were considered to have IMN in youth.

Of the 21 patients included in the study, the male to female incidence ratio was 3.2:1. Other study findings included NS (81\%), hypertension (14.3\%), microscopic hematuria $(51.7 \%)$, and renal dysfunction (9.5\%). The majority of the IMN cases included in this study were classified as either stages I or II, accounting for $95.23 \%$ of all the cases.

In this group, all 21 patients had mild proliferation of the glomerular mesangial cells and mesangial matrix. Mesangial cells have previously been reported to increase with the occurrence of IMN, and particularly when associated with focal segmental glomerulosclerosis (FSGS), the proliferation of mesangial cells is thought to be more evident (Lee et al., 2006; Gupta et al., 2010). In comparison to elderly patients, mesangial proliferation is high in young IMN patients, suggesting that the renal mesangial region of young patients is more sensitive to external stimuli.

Unlike in typical IMN patients, where immunofluorescence examination shows highintensity deposition of $\operatorname{IgG}$ and granular $\mathrm{C} 3$ on the subepithelium and basement membrane, in this study, in addition to IgG and C3, the 21 patients exhibited high-intensity deposition of a variety of other immune complexes (IgA, IgM, C1q, and FRA, among others) and at multiple sites (subepithelium, basement membrane, and mesangial region), while 14 patients even exhibited the phenomenon of "complete brightness". Clinically, in IMN cases associated with significant mesangial proliferation, positive immunofluorescence and multi-site deposition of electron-condensing substances are characterized as atypical membranous nephropathy, and these cases are often highly suspected as secondary membranous nephropathy. While this cannot be excluded in the present study because there was no other evidence of the condition being secondary in nature, the 21 patients included were diagnosed as having IMN.

Data indicate that a third of IMN patients gradually progress to end-stage renal disease (Valentini et al., 2009), but the factors affecting prognosis are varied in literature. In most cases, patients were elderly men with continuous massive proteinuria, and the pathological stages were mainly identified as stage III or IV accompanied by renal dysfunction at disease onset, and the progression of tubulointerstitial lesions, as well as poor prognosis associated with FSGS (Wasserstein, 1997; Valentini et al., 2009; Ponticelli and Passerini, 2010b; Praga and Rojas-Rivera, 2012). In the present study, pathological stages were identified as stage I in 16 cases, stage II in 6 cases, and stage III in 1 case. In the patient identified as being in stage III (accompanied by renal dysfunction, FSGS, moderate tubulointerstitial changes), complete remission was achieved after treatment. Among the 6 patients who showed significant tubulointerstitial changes, 3 patients obtained complete remission, 1 patient obtained partial remission. Among the 4 patients with NS and FSGS, 1 patient obtained complete remission simply after hormone therapy; in 1 patient, hormone therapy was ineffective for 12 weeks but the patient subsequently achieved complete remission with FK506. In 2 patients who had renal dysfunction, 1 patient obtained complete remission with simple hormone therapy. Taken 
together these findings indicate that for young IMN patients, the aforementioned pathological changes were not the absolute factors affecting prognosis.

IMN treatments include non-specific symptomatic and etiological treatments. Symptomatic treatments include anticoagulation therapy, lipid regulation, diuresis, anti-hypertensive medication, and proteinuria reduction with angiotensin-converting enzyme inhibitors/ angiotensin receptor blockers. Etiological treatments include immunosuppressive therapies such as glucocorticoids, cyclophosphamide, MMF, cyclosporin A, FK506, and azathioprine. Currently, the consistent view towards the treatment of IMN is that hormone therapy alone has a non-significant effect on IMN (Cattran et al., 1989; Beck et al., 2013), and many scholars advocate the combined use of immunosuppressant therapy (Ponticelli et al., 1998; Goumenos et al., 2006; Jha et al., 2007; Ponticelli, 2007; Dussol et al., 2008; Quaglia and Stratta, 2009; Kalliakmani et al., 2010; Polanco et al., 2010; Cattran et al., 2011; Howman et al., 2013). It is suggested that high-risk patients with deteriorated renal function should be given an active combination therapy of glucocorticoids and immunosuppressant, whereas in low-risk IMN patients, the adverse effects of glucocorticoid and immunosuppressant therapy should be avoided where possible (Quaglia and Stratta, 2009). The main study finding was that young IMN patients have higher complete remission rates with glucocorticoids, or a combination of glucocorticoids and immunosuppressant. A high overall remission rate also indicates that young IMN patients should be actively administered glucocorticoid and immunosuppressant therapy in the early stages in order to achieve complete remission. The sample size in this study, however, was small and further observation and investigation is still needed. Nevertheless, individualized treatment programs should be developed depending on the patient's condition, and the long-term prognosis should be taken into consideration.

\section{REFERENCES}

Beck L, Bomback AS, Choi MJ, Holzman LB, et al. (2013). KDOQI US Commentary on the 2012 KDIGO Clinical Practice Guideline for Glomerulonephritis. Am. J. Kidney Dis. 62: 403-441.

Cattran DC, Delmore T, Roscoe J, Cole E, et al. (1989). A randomized controlled trial of prednisone in patients with idiopathic membranous nephropathy. N. Engl. J. Med. 320: 210-215.

Cattran DC, Reich HN, Kim SJ and Troyanov S (2011). Have we changed the outcome in membranous nephropathy? A propensity study on the role of immunosuppressive therapy. Clin. J. Am. Soc. Nephrol. 6: 591-598.

du Buf-Vereijken PW, Branten AJ and Wetzels JF (2005). Idiopathic membranous nephropathy: outline and rationale of a treatment strategy. Am. J. Kidney Dis. 46: 1012-1019.

Dussol B, Morange S, Burtey S, Indreies M, et al. (2008). Mycophenolate mofetil monotherapy in membranous nephropathy: a 1-year randomized controlled trial. Am. J. Kidney Dis. 52: 699-705.

Ehrenreich T and Churg J (1968). Pathology of membranous nephropathy. Pathol. Ann. 3: 145-186.

Glassock RJ (2003). Diagnosis and natural course of membranous nephropathy. Semin. Nephrol. 23: 324-332.

Goumenos DS, Ahuja M, Davlouros P, El Nahas AM, et al. (2006). Prednisolone and azathioprine in membranous nephropathy: a 10-year follow-up study. Clin. Nephrol. 65: 317-323.

Gupta R, Sharma A, Mahanta PJ, Jacob TG, et al. (2010). Focal segmental glomerulosclerosis in idiopathic membranous glomerulonephritis: a clinico-pathological and stereological study. Nephrol. Dial. Transplant. 25: 444-449.

Howman A, Chapman TL, Langdon MM, Ferguson C, et al. (2013). Immunosuppression for progressive membranous nephropathy: a UK randomised controlled trial. Lancet 381: 744-751.

Jha V, Ganguli A, Saha TK, Kohli HS, et al. (2007). A randomized, controlled trial of steroids and cyclophosphamide in adults with nephritic syndrome caused by idiopathic membranous nephropathy. J. Am. Soc. Nephrol. 18: 1899-1904.

Kalliakmani P, Koutroulia E, Sotsiou F, Vlachojannis JG, et al. (2010). Benefit and cost from the long-term use of cyclosporine A in idiopathic membranous nephropathy. Nephrology 15: 762-767.

Lee BH, Cho HY, Kang HG, Ha IS, et al. (2006). Idiopathic membranous nephropathy in children. Pediatr. Nephrol. 21: 1707-1715. 
Li LS and Liu ZH (2004). Epidemiologic data of renal diseases from a single unit in China: analysis based on 13519 renal biopsies. Kidney Int. 66: 920-923.

Polanco N, Gutiérrez E, Covarsí A, Ariza F, et al. (2010). Spontaneous remission of nephrotic syndrome in idiopathic membranous nephropathy. J. Am. Soc. Nephrol. 21: 697-704.

Ponticelli C (2007). Membranous nephropathy. J. Nephrol. 20: 268-287.

Ponticelli C and Passerini P (2010a). Can prognostic factors assist therapeutic decisions in idiopathic membranous nephropathy? J. Nephrol. 23: 156-163.

Ponticelli C and Passerini P (2010b). Management of idiopathic membranous nephropathy. Expert Opin. Pharmacother. 11: 2163-2175.

Pontieelli C, Altieti P, Scolari F, Passerini P, et al. (1998). A randomized study compating methylprednisolone plus chlorambucil versus methylprednisolone plus cyelophosphamide in idiopathic membranous nephropathy. J. Am Soc. Nephrol. 9: 444-450.

Praga M and Rojas-Rivera J (2012). Glomerular disease: predicting outcomes in idiopathic membranous nephropathy. Nat. Rev. Nephrol. 8: 496-498.

Quaglia M and Stratta P (2009). Idiopathic membranous nephropathy: management strategies. Drugs 69: 1303-1317.

Reichert LJ, Koene RA and Wetzels JF (1998). Prognostic factors in idiopathic membranous nephropathy. Am. J. Kidney Dis. 31: 1-11.

Schieppati A, Mosconi L, Perna A, Mecca G, et al. (1993). Prognosis of untreated patients with idiopathic membranous nephropathy. N. Engl. J. Med. 329: 85-89.

Valentini RP, Mattoo TK, Kapur G and Imam A (2009). Membranous glomerulonephritis: treatment response and outcome in children. Pediatr. Nephrol. 24: 301-308.

Waldman M and Austin HA 3rd (2009). Controversies in the treatment of idiopathic membranous nephropathy. Nat. Rev. Nephrol. 5: 469-479.

Wasserstein AG (1997). Membranous glomerulonephritis. J. Am. Soc. Nephrol. 8: 664-674.

Wu HY (2002). Definition of youth age. China Youth Study 3: 36-39.

Xiong ZB, Yu XQ and Yang QQ (2004). Clinical and pathological features of idiopathic membranous nephropathy in 134 cases in Southern China area. Chin. J. Nephrol. 20: 26-29. 